\title{
$\mathrm{TPM}$ 활동요인의 효과에 대한 $\mathrm{AHP}$ 분석
}

\author{
연경화* \\ 청주대학교 경영학과 교수
}

\section{AHP Analysis for the effect of TPM Activity factors}

\author{
Yeon Keyong Hwa* \\ Dept. of Business Administration, Cheongju University ${ }^{*}$
}

\begin{abstract}
요 약 일반적으로 TPM 전개프로그램은 추진단계가 정하여져 있다. 그러나 생산시스템의 효율을 극대화시키기 위 해서는 TPM의 구체적인 추진요령이나 절차 등에 있어서 업종이나 생산방식, 설비의 종류와 상태, 당면하고 있는 문 제들이 서로 다르기 때문에 각각의 기업환경에 맞는 추진방식을 선택하여야 한다. 본 연구에서는 TPM을 추진하고 있는 제조업체를 대상으로 $\mathrm{KSA}$ 가 제시하는 TPM활동별로 2단계로 세분화하여 각 단계별 활동 중에서 어떠한 활동들 이 더 중요하다고 인식되고 있는지를 AHP방법을 이용하여 분석하였다. 분석결과 TPM추진의 1 단계 활동에 있어서는 개별개선을 가장 중요한 활동으로, 그리고 전체 활동 중에서 가장 중요한 활동으로는 현장불합리 개선 및 Loss 개선, 그 다음은 준비 및 $5 \mathrm{~S}$ 활동을 위한 기본조건 체계를 구축하는 활동, 초기 청소 등의 순서인 것으로 분석되었다.
\end{abstract}

주제어 : TPM, AHP, TPM활동, 개별개선

\begin{abstract}
Generally, TPM activity promotion program steps have been determined. However, in order to maximize the efficiency of the production system of TPM, industry types or production methods, equipment type and condition, the problems facing the environment are so different that tips and procedures should be chosen in the right way for each company to promote. This study is aimed at a manufacturing company promoting TPM KSA presented. The TPM activities are broken into two phases. And we employ the AHP method to analyze which phase of activities are more important to recognize than the other phase of activities. In Step 1 of the analysis of TPM activities, promotion improvement is the most important activities of the individual, and the overall activity of the most important activities are the field improvement and loss improvement, then followed by the basic conditions for the preparation and $5 \mathrm{~S}$ activities to build a system activity, such as the order of the initial cleaning was analyzed.
\end{abstract}

Key Words : TPM, AHP, TPM Activity, Individual Improvement

\section{1. 서론}

최근 기업경영의 특징은 기업의 성장과 변화에 따라
자산·서비스관리 솔루션의 효율적 운영의 중요성이 매 우 많이 강조되고 있다는 점이다. 즉, 기술발전으로 인한 생산시스템의 기계화·자동화의 정도가 고도화되고 제

\footnotetext{
* 본 연구는 2012 2013학년도에 청주대학교 경영경제연구소가

Received 9 November 2013 , Revised 10 January 2014

Accepted 20 February 2014

Corresponding Author: Yeon Keyong Hwa(Cheongju University)

Email: yknwao@nate.com
}

ISSN: 1738-1916
지원한 학술연구조성비(특별연구과제)에 의해 연구되었음.

(C) The Society of Digital Policy \& Management. All rights reserved. This is an open-access article distributed under the terms of the Creative Commons Attribution Non-Commercial License (http://creativecommons.otg/licenses /by-nc/3.0), which permits unrestricted non-commercial use, distribution, and reproduction in any medium, provided the original work is properly cited. 
조공정도 훨씬 더 복잡해졌으며, 이에 따른 설비투자액 도 증대되고 있다. 따라서 설비관리 및 효율적 운영이 기 업의 생산성향상과 원가절감에 밀접하게 연관되어 있고, 문제발생시 그 해결방안도 점자 진화하고 있는 상태이다. 이러한 측면에서 종합적 생산성관리는 단순한 설비의 보 전성 유지뿐만 아니라 보전성 향상을 위한 모든 요소, 즉 기업의 내·외적 모든 요소들을 포함한 총체적 능력이 관리대상으로 포함되는 접근방법으로 설비설계와 개발 등으로 생산보전을 확대시키고 있는 추세에 있다고 하겠 다. 이러한 추세는 설비유효성을 극대화라는 목표를 강 조하게 되었고, 그 목표달성을 위한 방법의 하나로 TPM 활동을 도입하게 된 것이라 할 수 있다.

이러한 중요성의 강조에 따라 본 연구에서는 TPM 활 동의 기초가 되는 활동과 각 단계별로 추진되는 활동들 의 중요성이 어떻게 인식되고 있는지를 파악하고자 한다. 즉, 10 개월 이상 TPM 교육을 받아온 생산현장의 실무자 들을 대상으로 설문조사를 실시하고, 조사된 내용을 바 탕으로 $\mathrm{AHP}$ 분석을 통해 각 단계별로 추진되는 활동들 에 대해 어느 정도 중요하다고 인식하는지 그 중요성의 정도를 파악하고자 한다. 왜냐하면 실무자별로 단계별 활동에 대한 인식이 다를 것이고, 따라서 추진되는 활동 들의 중요도에 대한 우선순위를 분석하는 것은 TPM활 동의 계획수립에 기초가 되는 정보를 제공할 수 있다고 판단하기 때문이다. 그러나 TPM 추진활동별 중요도에 관한 연구가 많이 진행되고 있지 않아서 연구에 어려움 이 따르지만 실무자들의 인식에 대해 연구의 초점을 맞 추고자 한다. 향후 본 연구는 TPM 활동의 추진에 보완 적인 지침이 될 수 있기를 기대하고 있다.

\section{2. 이론적 고찰}

\subsection{TPM의 개념}

TPM이란 Total Productive Maintenance(전원참여 생산보전), 또는 Total Preventive Maintenance(전원참 여 설비보전)의 약자로서 1971년 일본의 JIPM에서 제창 하였고, 한국에서 1980년대 중후반에 KSA가 처음으로 도입한 설비관리 혁신활동을 말한다.

$\mathrm{KSA}$ 에 의하면 TPM을 생산부문의 TPM에 대한 정의 에서 전사적 TPM으로 확대하여 (1) 생산 시스템 효율화
의 극한을 추구(종합적 효율화)하는 기업 체질 조성을 목 표로, (2) 생산 시스템의 라이프사이클 전체를 대상으로 한 '재해 제로, 불량 제로, 고장 제로' 등 모든 로스를 미 연 방지하는 구조를 현장, 현물로 구축하며, (3) 생산 부문 을 비롯하여, 개발, 영업, 관리 등 모든 부문에 걸쳐서, (4) 톱으로부터 제일선 종업원에 이르기까지 전원이 참가하 고, (5) 중복 소집단 활동에 의해 로스 제로를 달성할 것 등으로 정의하고 있다.[1]

이상의 정의에 따르면 중요한 것은 고장, 불량, 재해 등이 발생화지 않도록 사전에 예방하는 것이 필수조건이 라 할 수 있다. 그런데 제품의 생산에 있어서는 제조현장 에서의 설비가 중요한 역할을 하지만, 이 보다는 설비에 관련된 작업자, 보전요원 등 인적 자원의 중요성이 더욱 더 강조된다고 볼 수 있다. 왜냐하면 정상적인 상태를 유 지하기 위해서 평소에 청소, 점검, 급유, 나사 조이기, 정 도 체크 등을 통해서 노화를 방지하는 등의 활동은 인간 이 담당하기 때문이다. 특히 설비가 복잡해질수록 인적 자원의 역할에 대한 중요성도 점차 더 강조가 될 것이다. 따라서 생산시스템의 목표를 최상으로 달성하기 위해 가장 중요시해야 되는 목표는 현장의 체질을 개선하는 것이라 할 수 있다. 대부분의 제조현장은 작업자와 설비 의 대응관계, 즉 한 작업자가 한 대 혹은 여러 대의 설비 를 맡고 있으며 자동화설비, 트랜스 머신, 로봇 등 설비의 종류에 관계없이 작업자가 설비가 밀접하게 연결되어 움 직이는 man-machine system으로 이루어져 있기 때문이 다. 그러므로 시스템을 극대화시키기 위해서는 설비의 구성부품 등 설비의 성능을 항상 최고의 상태로 갖추어 야 하고 또 작업자, 보전요원 등 설비와 관련된 사람은 역할 분담을 명확하게 하여 설비의 상태를 장시간에 걸 쳐서 유지해야 하는 것이 필수라 하겠다.

\subsection{TPM 활동}

TPM 활동의 가장 큰 특색은 중복 소집단 활동에 의 한 운전요원 및 보전요원이 함께 하는 자주보전활동과 함께 예지보전 활동을 종합적으로 실시하여 설비관리를 전개하는 것이라 할 수 있다. 이러한 TPM 활동은 생산 부문을 대상으로 실시되어 왔으나 점차로 제품개발부문 과 관리측면인 간접부문까지 확대되어 전개되었다. 기본 적으로 $\mathrm{TPM}$ 의 적용은 이에 관계하는 인간의 사고방식 과 행동에 의존하고 있으며, 이는 5 행의 실천을 통해 다 
〈Table 1〉 5S/TPM Activity Promotion Steps and Method

\begin{tabular}{|c|c|}
\hline Activity & Promotion Steps and Method \\
\hline \multirow{7}{*}{$\begin{array}{l}\text { Autonomous } \\
\text { Maintenance }\end{array}$} & Initial cleaning \\
\hline & Sources difficult place countermeasures \\
\hline & Write a temporary basis Facilities Management \\
\hline & Equipment overhaul \\
\hline & Process overhaul \\
\hline & Autonomous maintenance systematization \\
\hline & Autonomous management \\
\hline \multirow{2}{*}{$\begin{array}{l}\text { Individual } \\
\text { Improvement }\end{array}$} & $\begin{array}{l}\text { Irrational field improvement and Loss improvement, Improve the system to achieve the goal of promoting } \\
\text { individual settlement }\end{array}$ \\
\hline & $\begin{array}{l}\text { Plant efficiency and analysis of inhibition of productivity in various Loss,, Develop individual improvement } \\
\text { theme activities for circles and middle managers }\end{array}$ \\
\hline \multirow{4}{*}{$\begin{array}{c}\text { Planned } \\
\text { Maintenance }\end{array}$} & Preparations and establish the basic conditions for the $5 \mathrm{~S}$ activity system \\
\hline & Restoring deteriorated, improve weaknesses and Base management system for MP Activity \\
\hline & Establish Information management system and regular preservation system \\
\hline & Building predictive maintenance systems and planned maintenance assessment \\
\hline \multirow{2}{*}{$\begin{array}{l}\text { Quality } \\
\text { Maintenance }\end{array}$} & Improved facilities for the source regions of Defective driving \\
\hline & The introduce and deployment Quality preservation activity for zero loss defective \\
\hline \multirow{3}{*}{$\begin{array}{l}\text { Education \& } \\
\text { Training }\end{array}$} & TPM Basic Course(company), TPM manual/maintenance textbooks training-Circle, field worker \\
\hline & TPM practical courses(company), total inspection manual training-propulsion staff \\
\hline & TPM application process(company), in charge of facility manual training-Administrator \\
\hline
\end{tabular}

져진 것으로 TPM의 본격적인 추진의 전단계로서 이 5행 을 전사적으로 실천하는 것이 효과적인 것으로 나타나고 있다. $5 \mathrm{~S}$ 는 $\mathrm{TPM}$ (전사적 생산보전)운동의 기본을 이루는 활동으로서 직장내의 각 부문 등에 있어 모든 낭비 요소 를 배제하여 최대의 효율을 높이는 데 그 의의가 있다. 또한 $5 \mathrm{~S}$ (정리, 정돈, 청소, 청결, 생활화)를 발전 심화시킴 으로써 효율이 높은 직장, 깨끗한 직장, 보람있는 직장을 만드는데 그 목적이 있다[2].

일반적으로 TPM은 기업내의 전 조직이 참여하는 설 비계획, 설비사용, 설비지원부문의 종합적 활동으로 보통 8대 기능 추진 핵심 포인트가 제시되고 있지만, 본 연구에 서는 생산현장의 실무자들이 TPM활동에 대해 어떻게 인 식하고 있는가를 파악하는 것이 핵심이라 할 수 있다. 따 라서 한국표준협회 TPM 생산혁신팀에서 요약, 정리하여 제시하고 있는 8가지의 TPM활동 중에서 현장에서의 중 요성이 비교적 덜 인식된다고 판단하는 활동은 조사에서 제외하기로 하고 [Table 1]과 같이 5가지 활동에 국한해 서 연구에 적용하기로 한다.[3] [Table 1]에서 요약, 정리 한 활동을 조금 더 구체적으로 살펴보면 다음과 같다.
(1) 자주보전 : 자주보전활동은 제조부문을 중심으로 한 오퍼레이터의 활동이다. 설비의 기본조건(청소, 급유, 더 조이기)을 정비하여 이를 유지하고 사용조건을 지키 며, 총점검에 의해 열화를 복원하고 설비에 강한 오퍼레 이터를 만들어 자주관리 한다는 목표를 7스텝의 전개 프 로그램에 근거한 교육/훈련과 실천의 반복으로 실현, 즉 작업자 스스로가 정한 기준에 따라 행동하는 설비의 유 지관리 활동을 자주보전이라고 한다[4].

(2) 개별개선 : 설비, 공정 등 정해진 대상에 대하여 철 저한 Loss의 배제와 성능향상을 추구하여 최고의 효율을 이룩하기 위한 개선 활동을 말한다. 이 활동은 Loss의 크 기나 난이도에 따라 팀을 구성하여 원인에 대한 물리적, 논리적인 원리를 근거로 근본 원인을 해결하는 특성을 지니고 있기 때문에 테마의 선정이 매우 중요한 것으로 인식되고 있다. 즉, 효율이 낮고, 수평전개 요소가 큰 공 정, 또는 Neck 공정, Loss가 큰 공정, 회사의 방침과 부합 되는 공정 등 어떠한 테마를 선정하느냐에 따라 성과가 좌우되기 때문이다.

(3) 계획보전 : 설비의 성능을 최대한으로 유지하고 수 
명연장과 생산능력을 향상시키기 위하여 보전부문을 중 심으로 설비의 설계, 설치, 운전, 폐기까지 설비의 전 생 애를 통하여 보전비용을 최소화하고 설비열화에 의한 손 실을 낮추는 활동을 말한다.[5] 즉, 설비의 최적보전주기 와 구성품의 최적교환기를 이론 또는 경험값에 의해 설 정하여, 주기가 되면 무조건 수리하거나, 설비를 정기적 으로 분해·점검하고 불량인 것을 교환하는 예방보전, 개 량보전, 사후보전 체제를 구축하는 활동을 말한다.

(4) 품질보전 : 품질불량 제로를 위한 보전, 구체적으로 는 $100 \%$ 양품의 상태를 유지하기 위해 불량 제로의 조건 을 설정하고, 그 조건을 시계열적으로 점검·측정하여 그 측정치를 기준치 이내로 조절함으로써 품질불량을 예방 하는 한편, 측정치의 추이를 관찰함으로써 품질불량 발 생가능성을 예지하여 사전에 대책을 수립하는 활동이라 할 수 있다.[6] 이는 설비가 점차 자동화·고급화되어가는 추세에 따라 효과적인 설비보전이 고수준의 품질유지 및 향상을 이룰 수 있게 되어 품질보전의 중요성의 증대로 도입된 활동이라 할 수 있다.

(5) 교육훈련 : 운전원 및 보전요원들로 하여금 업무나 활동에 필요한 지식과 방법을 익히도록 하고 이러한 지 식과 방법을 실천, 실습을 통해 몸에 익숙토록 함으로써 설비에 강한 사람을 만들기 위해서 하는 활동이다. 교육 훈련은 1 단계로 사내교육을 통한 보전기초 교재개발 및 교육, 2 단계로 설비의 범용적인 기기 총점검 매뉴얼의 개 발 및 교육, 3 단계로 담당 공정 및 설비(생산, 환경, 유틸 리티설비를 말함)중심의 설비매뉴얼 개발 및 교육 등의 단계로 이루어져야 효과적인 기능교육이 될 수 있는 것 으로 언급되고 있다.[7]

\section{3. $\mathrm{AHP}$ 분석}

\section{1 연구 설계}

Saaty에 의하여 개발된 $\mathrm{AHP}$ (Analytic Hierarchy Process) 기법은 다수의 속성들을 분류하여 각 속성의 중 요도를 파악함으로써 최적 대안을 선택하는 기법이다[8]. $\mathrm{AHP}$ 는 양적, 질적 자료 모두를 포함하는 복잡한 여러 기 준의 의사결정 문제를 해결하기 위해 사용되는데, 복잡 한 문제는 계층적 구조를 갖는 하위의 문제(subproblem)로 분류할 수 있어, 계층적 구조의 틀을 형성한
다는 기본적 원리를 바탕으로 분석이 가능하다. $\mathrm{AHP}$ 는 이미 언급한 것처럼 의사결정의 전과정을 여러 단계로 나눈 후 이를 단계별로 분석하여 최종적인 의사결정에 이르는 방법으로 6단계로 진행된다[9].

첫째, 가장 기본적인 목표를 제 1 수준으로 하여 계층적 구조를 설정한다. 둘째, 전문가나 의사결장자로부터 정성 적 대안을 쌍대 비교할 수 있는 척도로 각 계층구조에 일 치하는 자료를 수집한다. 셋째, 두 번째 단계에서 얻은 자 료를 다양한 기준으로 쌍대 비교할 수 있도록 행렬형태 로 변환한다. 넷째, 다양한 기준에 따라 상대적 중요도를 얻기 위한 비교행렬에서 표준화된 주 고유벡터에 일치하 는 아이겐 값을 산출한다. 다섯째, 일관성 지수를 산출하 게 되며 이를 이용하여 의사결정자의 논리적 일관성 유 지여부를 확인한다. 일반적으로 일관성지수를 Random Index로 나눈 비율이 0.1을 넘게 되면 의사결정자가 논리 적 일관성을 잃고 있는 것으로 판단하여 의사결정을 재 검토하는 기준으로 하고 있다. 여섯째, 각 대안별 비율에 하위수준의 비율을 곱해서 전체 목표의 비율을 얻는다.

또한 $\mathrm{AHP}$ 는 계층구조를 구성하고 있는 요인들간의 쌍대비교에 의한 평가자의 지식, 경험 및 직관을 포착하 고자 하는 방법론이라 할 수 있다. 쌍대 비교를 위해서 비교척도를 사용하는 데, 다음의 [Table 2]와 같은 범위를 사용한다[10].

\section{〈Table 2〉 Pairwise comparison Scale for AHP}

\begin{tabular}{|c|c|}
\hline Meaningful evaluation measure between items & score \\
\hline \hline extreme importance of one element over another & 9 \\
\hline very strongly favored one activity over another & 7 \\
\hline strongly favor one activity over another & 5 \\
\hline slightly favor one activity over another & 3 \\
\hline two activities contribute equally to the objective & 1 \\
\hline
\end{tabular}

위 [Table 2]에 제시된 바와 같은 척도로 쌍대비교를 위한 행렬을 작성하기 위해 [Table 3]과 같은 설문을 이 용하기로 하였다. 설문조사의 대상은 현재 자동차 부품 을 생산하는 기업체에서 생산활동에 종사하고 있는 작업 자들 중 10개월 이상 TPM교육을 받은 9명을 대상으로 하였다. 조사는 [Table 3]에서 보듯이 각 평가변수별로 각 계층의 요소들을 쌍으로 Activity1과 Activity2를 비 교할 때 Activity1이 Activity2보다 더 중요하다면 1을 중 심으로 Activity1 방향인 왼쪽의 숫자에 표시를 하고, 반 
대로 Activity2가 더 중요하다면 1을 중심으로 Activity2 의 방향인 오른쪽의 숫자에 표시하도록 설명을 한 후 조 사를 실시하였다. 이렇게 수집된 자료는 Expert Choice (이하 EC로 표기) 11.5 를 이용하여 분석을 실시하였다.

〈Table 3〉 Pairwise comparison Rating Scale

\begin{tabular}{|c|c|c|c|c|c|c|c|c|c|c|c|c|c|c|c|c|c|c|}
\hline \multirow{2}{*}{$\begin{array}{c}\text { evaluation } \\
\text { variable }\end{array}$} & \multicolumn{17}{|c|}{ Scale } & \multirow{2}{*}{$\begin{array}{c}\text { evaluation } \\
\text { variable }\end{array}$} \\
\hline & \multicolumn{7}{|c|}{ absolutely } & \multicolumn{2}{|c|}{ equal } & & & \multicolumn{6}{|c|}{ absolutely } & \\
\hline Activity1 & 9 & 8 & 7 & 6 & 5 & 4 & 3 & 2 & 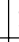 & & & 45 & 5 & 6 & 7 & 8 & & Activity2 \\
\hline Activity1 & 9 & 8 & 7 & 6 & 5 & 4 & 3 & 2 & & & 3 & 4 & 5 & 6 & 7 & 8 & 9 & Activity3 \\
\hline Activity2 & 9 & 8 & 7 & 6 & 5 & 4 & 3 & 2 & & & & 4 & 5 & 6 & 7 & 8 & & Activity3 \\
\hline Activity2 & 9 & 8 & 7 & 6 & 5 & 4 & 3 & 2 & & & & 4 & 5 & 6 & 7 & 8 & & Activity4 \\
\hline$\vdots$ & 9 & 8 & 7 & 6 & 5 & 4 & 3 & 2 & & & & $4: 5$ & 5 & 1 & 7 & 8 & 9 & $\vdots$ \\
\hline
\end{tabular}

\subsection{AHP 분석}

본 연구에서 해결하고자 하는 문제와 연구모형은 [Table 1]에서 제시한 바와 같이 2단계의 활동으로 1단계 는 5 가지 활동과 2 단계는 1 단계의 각 활동별 세부활동으
로 구성하였다. 이렇게 구성된 계층적 구조를 분석도구 인 $\mathrm{EC}$ 의 모형구축에 동일하게 설정하고, 각 단계별 활동 에 설문조사로 수집된 자료를 입력한 후 분석하였으며, 분석한 결과를 요약, 재정리한 것이 다음의 [Table 4], [Table 5]와 같다.

이미 언급한 것처럼 본 연구에서는 TPM 활동에 매우 익숙한 9명을 대상으로 설문을 이용하여 자료를 수집하 였으나, 분석에 사용한 $\mathrm{EC} 11.5$ 는 참여자가 3 인용이기 때 문에 1 회 3 인의 자료를 입력하여 자료를 분석하고, 동일 한 방법으로 3 회를 반복하여 9 명의 자료를 전부 분석에 투입하였다.

이와 같은 방법으로 분석하여 출력된 결과를 정리한 [Table 4]는 참석자별, 각 단계별 비일관성비율을 나타낸 것이다. 비일관성비율은 판단의 논리적인 모순을 측정하 는 척도로 판단 자체에서 생기는 비일관성뿐만 아니라 판단하는 과정에서 발생할 수 있는 오류를 확인하는 데 도 유용한 것으로, 일반적으로 비일관성비율은 0.1 보다

〈Table 4〉 Inconsistency Table

\begin{tabular}{|c|c|c|c|c|c|c|c|}
\hline Name & Overall & $\begin{array}{c}\text { Goal:TPM } \\
\text { Activity }\end{array}$ & $\begin{array}{l}\text { Autonomous } \\
\text { Maintenance } \\
\text { (L: .092 G: .092) }\end{array}$ & $\begin{array}{l}\text { Individual } \\
\text { Improvement } \\
\text { (L: .549 G: .549) }\end{array}$ & $\begin{array}{l}\text { Planned } \\
\text { Maintenance } \\
\text { (L: .281 G: .281) }\end{array}$ & $\begin{array}{l}\text { Quality } \\
\text { Preservation } \\
\text { (L: .039 G: .039) }\end{array}$ & $\begin{array}{l}\text { Education and } \\
\text { Training } \\
\text { (L: .039 G: .039) }\end{array}$ \\
\hline & \#Factors & 5 & 7 & 2 & 4 & 2 & 3 \\
\hline P2 & .0925 & .0971 & .0838 & .0000 & .0760 & .0000 & .0896 \\
\hline P3 & .0987 & .1124 & .0829 & .0000 & .0522 & .0000 & .0618 \\
\hline P4 & .0649 & .0691 & .0907 & .0000 & .0336 & .0000 & .0511 \\
\hline
\end{tabular}

〈Table 5〉 AHP Analysis Results

\begin{tabular}{|c|c|c|c|}
\hline Step 1 & Step 2 & Total weight & Rank \\
\hline \multirow{7}{*}{$\begin{array}{c}\text { Autonomous Maintenance } \\
\text { (.0.092) }\end{array}$} & Initial cleaning $(0.538)$ & .072 & 3 \\
\hline & Sources difficult place countermeasures $(0.187)$ & .025 & 10 \\
\hline & Write a temporary basis Facilities Management $(0.080)$ & .011 & 11 \\
\hline & Equipment overhaul $(0.058)$ & .008 & 12 \\
\hline & Process overhaul $(0.049)$ & .006 & 15 \\
\hline & Autonomous maintenance systematization $(0.040)$ & .005 & 16 \\
\hline & Autonomous management $(0.049)$ & .007 & 13 \\
\hline \multirow{2}{*}{$\begin{array}{l}\text { Individual Improvement } \\
(0.549)\end{array}$} & Irrational field and Loss improvement $\quad(0.896)$ & .428 & 1 \\
\hline & Loss Analysis of facility efficiency $(0.104)$ & .049 & 4 \\
\hline \multirow{4}{*}{$\begin{array}{l}\text { Planned Maintenance } \\
(0.281)\end{array}$} & Establish the basic conditions for the $5 \mathrm{~S}$ system $(0.697)$ & .219 & 2 \\
\hline & Base management system for MP Activity $\quad(0.129)$ & .028 & 8 \\
\hline & Establish regular preservation system $(0.091)$ & .026 & 9 \\
\hline & Building predictive maintenance systems $(0.083)$ & .040 & 5 \\
\hline \multirow{2}{*}{$\begin{array}{l}\text { Quality Maintenance } \\
\qquad(0.039)\end{array}$} & Improvement of the source of defects $(0.874)$ & .030 & 6 \\
\hline & Develop Quality preservation activity $(0.126)$ & .004 & 17 \\
\hline \multirow{3}{*}{$\begin{array}{l}\text { Education and Training } \\
(0.039)\end{array}$} & TPM Basic Course-Circle, field worker (0.745) & .030 & 7 \\
\hline & TPM practical Course-propulsion staff $\quad(0.183)$ & .007 & 14 \\
\hline & TPM application Course-Administrator $\quad(0.072)$ & .003 & 18 \\
\hline
\end{tabular}




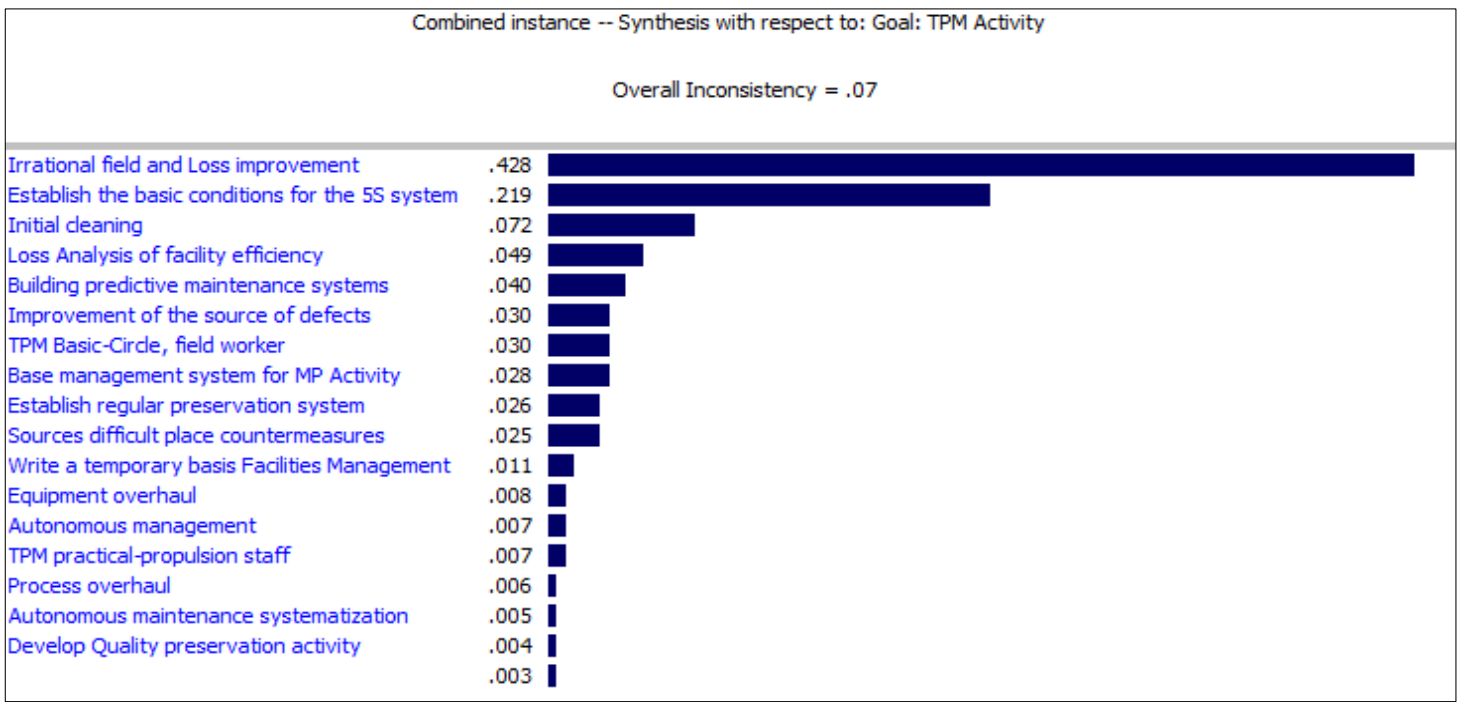

[Fig. 1] AHP Synthesis Results

작아야 논리적 일관성을 유지하는 상태라 할 수 있 다.[11] 분석결과 [Table 4]에서 보는 것처럼 각각 3명씩 통합하여 계산된 P2, P3, P4에 있어서 P3 그룹의 TPM활 동의 경우를 제외하고 전반적인 비일관성비율은 0.1 이하 로 나타났다.

또한 분석결과로 나타난 각 활동별 중요성의 우선순 위를 [Table 5]와 같이 재정리하여 요약하였다. 이 표에 의하면 1 단계 활동에 대해서 인식하고 있는 중요성의 우 선순위는 개별개선(0.549), 그 다음은 계획보전(0.291), 자 주보전(0.092) 등인 것으로 확인할 수 있었으며, 품질보 전(0.039)과 교육훈련(0.039)은 중요성의 정도를 가장 낮 게 인식하고 있는 것으로 확인할 수 있었다. 그리고 자주 보전활동에 있어서는 초기청소(0.539)가 가장 중요한 것 으로 나타났으며, 개별개선에서는 현장에서의 불합리 및 Loss 개선활동(0.896)을 가장 중요하게 인식하는 것으로 나타났다. 그리고 계획보전에서는 준비 및 $5 \mathrm{~S}$ 활동을 위 한 기본조건 체제구축(0.697)을 가장 중요하게, 품질보전 에서는 불량발생원에 대한 설비부위의 개선(0.874)을 보 다 중요한 것으로, 교육훈련에 있어서는 분임조활동 및 현장 실무자를 위한 TPM 매뉴얼에 의한 교본교육 (0.745)을 보다 중요하게 인식하는 것으로 파악되었다.

[Fig.1]은 단계 0 수준인 전체의 목표와 관련하여 각 활동들을 비교분석한 결과를 출력한 것이다. 그림에서 보는 바와 같이 9 명의 자료를 분석한 전체 18 개 활동의
비일관성비율도 0.07 이하로 나타나 조사에 참여한 작업 자들의 논리적 일관성은 유지되는 것으로 판단할 수 있 었다. 이 분석결과에 의하면 TPM을 추진함에 있어서 가 장 중요한 것으로 인식하고 있는 활동은 현장에서의 불 합리 및 Loss 개선활동(0.428)인 것으로 나타났고, 그 다 음으로 준비 및 $5 \mathrm{~S}$ 활동을 위한 기본조건 체제구축 (0.219), 초기청소(0.072) 등의 순위로 각 활동의 중요성 이 인식되고 있음을 알 수 있었다. 이처럼 $\mathrm{AHP}$ 기법을 이 용한 분석결과 TPM 활동을 도입, 추진하기 위해서는 어 떠한 활동들을 중심으로 진행하여야 할 것인지를 파악하 기가 용이하게 그 결과를 제공하고 있다.

\section{4. 결론}

본 연구에서는 TPM 활동을 추진하는데 있어서 단계 별 활동들의 중요성이 어떻게 인식되고 있는지를 파악하 기 위한 목적으로 진행되었다. 이를 위해 고자 TPM활동 에 대한 이론들을 종합하고, 한국표준협회에서 제시하고 있는 추진단계별 활동 중에서 생산현장에서 실무자들이 직접 담당하게 될 비중이 높은 5 개 활동을 대상으로 계층 적 구조모형을 설정하고 $\mathrm{AHP}$ 기법을 이용한 분석을 실 시하였다. 그리고 분석을 위한 자료는 TPM을 도입하기 위해 준비 및 교육을 10 개월 이상 계속해서 진행하고 있 
는, $\mathrm{CNC}$ 등의 자동화 기계를 사용하여 자동차부품을 생 산하는 중소기업의 경험이 풍부한 현장실무자를 대상으 로 하여 수집하였다.

분석결과 설비가 지니고 있는 기능이나 성능을 최고 로 발휘할 수 있도록 효율화를 저해하는 현장의 불합리 한 부분을 개선하거나 Loss를 철저하게 제거하는 개별개 선활동을 가장 중요하게 인식하는 것으로 나타났다. 그 리고 이러한 활동이 가능하도록 설비에 대한 평가 및 고 장발생이나 설비의 약점을 개선할 수 있는 정보관리와 정기보전 체계를 확립하여 설비의 예방 또는 개량 등 밀 계획을 수립하여 보전을 수행하는 계획보전을 중요한 활 동으로 인식하고 있음이 파악되었다. 그러나 중요한 것 은 이와 같은 활동은 사람이 담당하는 것이므로 설비와 공정의 개선을 통해 사람의 체질을 바꾸는 것이고 궁극 적으로는 기업의 체질을 개선하는 것이며, 전원이 참여 한다는 의식이 가장 중요한 요소라 할 수 있다.

본 연구를 수행함에 있어서 한계점은 TPM도입 초기 단계의 실무자들을 대상으로 하였기 때문에 TPM활동을 추진함에 있어서 표준처럼 정해져 있는 초기단계의 활동 들이 중요하게 인식되었다는 점이다. 따라서 이미 TPM 활동이 활발하게 진행되고 있는 경우 각 단계별 활동에 대한 중요성의 우선순위는 다를 것인지를 파악해 볼 필 요가 있다는 점과, 이러한 우선순위의 파악에 대한 연구 가 부족하여 연구의 타당성을 뒷받침할 근거가 부족하다 는 점이다. 향후 다양한 연구를 통해 TPM 활동의 추진 에 보완적인 지침이 될 수 있는 방안을 마련하는데 초석 이 될 수 있기를 연구의 성과로 기대하고 있다.

\section{ACKNOWLEDGEMENTS}

This research was supported by subsidy from scientific research of Cheongju University Research Institute of Business and Economics in 2012-2013 school year.

\section{REFERENCES}

[1] KSA, http://www.q-korea.net/qmanage/qmanage_ process /qmanage_p_produce/tab3/20040911/1_21047.jsp [2] Anthony Kelly, "The Uses and Limitation of Total Productive Maintenance", Journal of Maintenance, October 1993 ; Nakajima, S., "Introduction to TPM", Productivity Press, 1988 pp.143-177.

[3] KSA, http://www.tpm.or.kr/business/business01_ 01.asp

[4] Blanchard, B., Verma, D. and Peterson, E., Maintenance ability, John Wiley \& Sons, Inc., New york, 1995, pp. 67-87

[5] KSA, Focused improvement practices for maximizing plant efficiency, 1994, pp. 2-5

[6] Ohwoon Kwon, Quality maintenance for quality defect-zero. http://www.q-korea.net/qmanage/qmanage_ process/qmanage_p_produce/tab3/20040930/1_21079.jsp

[7] KSA, Skill improve education/training for operators and maintenance men, http://www.q-korea.net/qmanage/ qmanage_process/qmanage_p_produce/tab3/2004093 0/1_21075.jsp

[8] Thomas L. Saaty, "Axiomatic Foundation of the nalytic Hierarchy Process, The Institute of Management Sciences", Management Science Vol.32, No.7, July 1986, pp. 841-844.

[9] Navneet Bhushan, Kanwal Rai(2004), "Strategic Decision Making-Applying the Analytic Hierarchy Process", Springer-Verlag London Limited, pp.15-17 1986, p. 843

[10] Dennis McCarthy, DR. Nick Rich, Lean TPM; A Blueprint for Change, Elsevier Ltd., 2004, pp. 20-21

[11] Expert Choice, Expert Choice 11-Quick start guide and tutorials-Part II:Tutorials, Expert Choice, Inc., 2004, p. 22

\section{연 경 화(Yeon, Keyonghwa)}

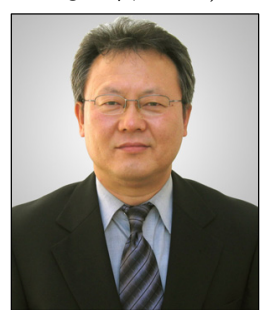

· 1993년 8월 : 청주대학교 경영학과 (경영학박사)

- 1995년 4월 현재 : 청주대학교 경 영학과 교수

- 관심분야 : $\mathrm{SCM}, \mathrm{TOC}, \mathrm{AHP}$, 통계 분석

•E-Mail : yknwao@nate.com 